\title{
Carboplatin/Nab- Paclitaxel/Pembrolizumab Regimen
}

National Cancer Institute

\section{Source}

National Cancer Institute. Carboplatin/Nab-Paclitaxel/Pembrolizumab Regimen. NCI

Thesaurus. Code C157619.

A reg imen consisting of carboplatin, nab-paclitaxel, and pembrolizumab that can be used in the treatment of metastatic squamous non-small cell lung cancer (NSCLC). 